\title{
ANÁLISE ESPACIAL MULTIVARIADA APLICADA NA AVALIAÇ̃̃O DA FERTILIDADE DO SOLO
}

\author{
Rodrigo Lilla Manzioneㄹ, Célia Regina Lopes Zimback²
}

\begin{abstract}
RESUMO
A variabilidade espacial de atributos do solo pode sofrer influências de diversos fatores, relativos à formação do solo: (material de origem, topografia, vegetação, clima e tempo), práticas de manejo (tipo de preparo do solo, rotação de culturas e adubação) e erosão. Nesse sentido, são necessárias técnicas apropriadas para acessar informações relativas a quais variáveis exercem maior influência sobre determinados aspectos do solo, a fim de aperfeiçoar o manejo e as práticas de correção de acidez e adubação, em propriedades agrícolas. O objetivo deste trabalho foi demonstrar a aplicação de técnicas geoestatísticas multivariadas na investigação do comportamento de um conjunto de variáveis químicas do solo. O trabalho foi realizado em uma área experimental de 70 hectares, no município de em Araguari$\mathrm{MG}$, onde foram analisadas as variáveis Fósforo (P), Potássio (K) e Matéria Orgânica (MO), sob o escopo do modelo linear de corregionalização. Nesse método, são ajustados $\mathrm{p}(\mathrm{p}+1)$ variogramas diretos e cruzados, para as variáveis, posteriormente decompostas em componentes principais. Os resultados demonstraram uma maior influência espacial da variável P, em micro e média escala, e uma influência dividida entre as varáveis $\mathrm{P}$ e $\mathrm{K}$, em longa escala. Devido ao manejo a que vem sendo submetida, a área apresenta uma fraca influência da MO, no comportamento conjunto dessas três variáveis do solo, e uma maior dependência espacial ligada às variáveis $\mathrm{P}$ e $\mathrm{K}$, durante as adubações para plantio de soja e milho. Os resultados indicam ainda um limite mínimo de 320,18 metros para criação de zonas de manejo desses elementos, em sistemas de adubação à taxa variável
\end{abstract}

Palavras-chave: geoestatística multivariada, modelo linear de corregionalização, agricultura de precisão.

\section{ABSTRACT \\ USE OF MULTIVARIATE SPATIAL ANALYSIS TO EVALUATE SOIL FERTILITY}

The spatial variability of soil attributes can be influenced by several factors such as soil formation (parent material, topography, vegetation, climate, and time), cultural practices (tillage, crop rotations, and fertilization), and erosion. Therefore, appropriate techniques are required to identify the variables that affect certain soil processes to improve soil management, liming and fertility practices in a farm. This study was done to demonstrate the applicability of multivariate geostatistics techniques to investigate the behavior of Phosphorus (P), Potassium (K) and Organic Matter $(\mathrm{OM})$ in a 70 hectares experimental area in Araguari, Minas Gerais State, Brazil. These soil constituents were analyzed with the use of linear coregionalization model. Direct and cross $p(p+1)$ variograms are adjusted, and then fractioned into principal components. The results showed a larger spatial influence of the variable $\mathrm{P}$ at micro and middle scale and shared influence of variables $\mathrm{P}$ and $\mathrm{K}$ at long scale. Due to the agronomical practices the influence of OM was small in the combined behavior of these constituents. $\mathrm{P}$ and $\mathrm{K}$ showed higher spatial dependence because of continuous applications of these fertilizers for soybean and corn cultivation. The results established a minimum limit of 320.18 meters for delimitating management zones for variable rate fertilizer zones.

Keywords: multivariate geostatistics, linear coregionalization model, precision agriculture.

\section{Recebido para publicação em 22/10/2010. Aprovado em 24/02/2011.}

1- Eng. Agrônomo, Professor assistente doutor. UNESP/Ourinhos, email: manzione@ourinhos.unesp.br;

2- Eng. Agrônomo, Professor livre docente. UNESP/FCA-Botucatu, email: czimback@gmail.com. 


\section{INTRODUÇ̃̃O}

A variabilidade dos solos tem sido abordada pela classificação numérica, por métodos de estatística multivariada, classificação contínua fuzzy, geoestatística, métodos de fractais, morfologia matemática e teoria do caos (BURROUGH et al., 1994). Embora estes métodos estatísticos permitam inferir sobre a variabilidade do solo (vertical e horizontal), a dependência espacial entre as amostras somente pode ser modelada por meio de análises geoestatísticas (WEBSTER, 1985). Esse tipo de análise está fundamentado na teoria das variáveis regionalizadas, que utiliza o variograma como estimador da dependência espacial, como descrito em diversos livros de referência (JOURNEL \& HUIJBREGTS, 1978; GOOVAERTS, 1997; VIEIRA, 2000; WACKERNAGEL, 2003).

A geoestatística tem-se mostrado de grande utilidade, na ciência do solo, para caracterizar e mapear a variabilidade espacial de suas propriedades. Assim, ao estudar-se a fertilidade de um solo, a partir de uma amostragem detalhada e de análises de solo georreferenciadas, pode-se fazer um levantamento completo do estado físico e químico da área, e caracterizar sua variabilidade espacial por meio de variogramas. Por fim, métodos de interpolação, como a krigagem, definem o grau de dependência no espaço da grandeza medida e o domínio de cada amostragem, baseados nesses variogramas, possibilitando um mapeamento que incorpora o sentido físico do fenômeno estudado aos mapas resultantes.

Todavia, os atributos do solo possuem interações entre si, além de apresentarem mudanças contínuas e gradativas pelo terreno, o que indica a geoestatística multivariada como análise alternativa, na análise conjunta de variáveis do solo. A modelagem de corregionalização das variáveis usa o denominado modelo linear de corregionalização (WACKERNAGEL, 2003) para todos os $p(p+1) / 2$ variogramas diretos e cruzados, das $p$ variáveis envolvidas no fenômeno em estudo, modeladas por uma combinação linear dos variogramas padronizados para um mesmo alcance. Nesta modelagem, supõe-se que o comportamento espacial das variáveis é o resultado da interação de diferentes processos, atuando, independentemente, a diferentes escalas espaciais.

Uma única amostra de solo, quando analisada em laboratório, fornece diversas informações sobre variáveis que revelam o estado nutricional de um determinado ponto ou parcela. Um conjunto de amostras, quando analisado, possibilita que se extraiam informações específicas de determinados conjuntos de variáveis, como macronutrientes, micronutrientes ou mesmo acidez do solo, que influenciam processos específicos no solo. A análise da estrutura de correlações entre as variáveis, levando em consideração as diferentes escalas, com a aplicação da análise das componentes principais, resume as relações entre as variáveis a cada escala espacial. A estimação das relações entre os fatores regionalizados e variáveis, como componentes espaciais, a diferentes escalas, por cokrigagem, para finalmente mapeá-los, seria o passo final (CASTIGNANÒ et al., 2000).

A realização de análises sobre conjuntos de variáveis específicos, para determinados fenômenos e processos do solo, permite que se obtenham informações sobre a variabilidade espacial desses atributos, com a agilidade necessária para que sejam consideradas nas propriedades agrícolas, ao planejarem seus esquemas de adubação, por exemplo, a tempo de atenderem aos propósitos agronômicos na delimitação de zonas de manejo da fertilidade do solo, dentro do calendário agrícola.

O presente artigo apresenta uma metodologia, para análise conjunta da estrutura espacial de conjuntos de variáveis, para estudos ligados à fertilidade do solo. Nesse estudo, analisou-se a corregionalização das variáveis Fósforo (P), Potássio (K) e Matéria Orgânica (MO), a partir de análises químicas georreferenciadas de solo, a fim de determinar com maior precisão seu comportamento no solo, em diferentes escalas espaciais, e fornecer informações que auxiliem na delimitação de zonas de manejo para adubação da cultura da soja.

\section{MATERIAL E MÉTODOS}

A área de estudo compreende um campo de produção de grãos, de 70 hectares, cultivados em rotação com soja e milho, em uma propriedade localizada no município de Araguari - MG, nas 
coordenadas $18^{\circ} 40^{\prime} \mathrm{S}$ e $48^{\circ} 15^{\prime} \mathrm{O}$. O relevo regional é medianamente dissecado em formas convexas, associadas a formas tabulares amplas, com drenagem pouco entalhada, pertencendo a área ao Planalto Setentrional da Bacia do Paraná. Apresenta relevo regional de plano a suave ondulado, vegetação de cerrado tropical e solo classificado, de acordo com EMBRAPA (1999), como Latossolo Vermelho distrófico, com textura argilosa, originários de cobertura laterítica Terciária e Quartenária indiferenciadas.

Para o estudo, foram coletadas amostras de solo a $20 \mathrm{~cm}$ de profundidade, com trado tipo Holandês, nas intersecções de uma grade quadrada sistemática de 60 metros, adensada para 30 metros em cinco pontos aleatórios (WOLLENHAUPT, 1995). Na Figura esta apresentado o esquema de amostragem na área de estudo. No total, foram coletadas 204 amostras de solo, identificadas com suas respectivas posições geográficas com sistema de posicionamento global (GPS). Posteriormente, as amostras foram analisadas, segundo métodos descritos pela EMBRAPA (1997). Foram realizadas análises químicas completas, para macro e micronutrientes.

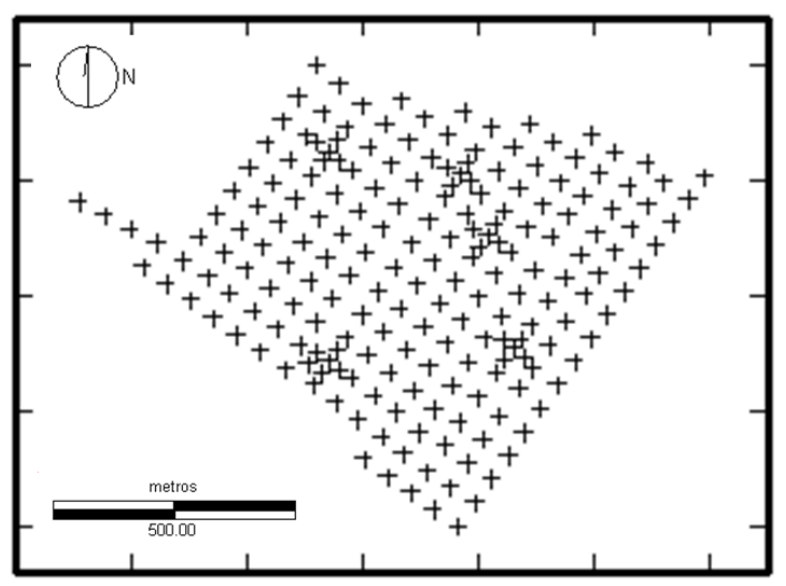

Figura 1. Esquema de amostragem, aplicado na área experimental de Araguari-MG.

Os dados de cada elemento ou parâmetro em estudo passaram por uma análise estatística exploratória, verificando-se características quantitativas e as respectivas distribuições de frequência em histogramas, segundo Gomes (1976). Para verificar a dispersão desses dados, foram examinados os quartis superiores e inferiores das frequências, identificando possíveis dados discrepantes (outliers), segundo Hoaglin et. al. (1992). Para que fossem atendidas as hipóteses geoestatísticas de estacionariedade dos dados, verificou-se a normalidade do conjunto de dados, segundo o teste de D'Agostino, descrito em Parkin \& Robins (1992). Fez-se também uma padronização dos dados, dividindo-se os valores pela respectiva média (BLACKMORE, 2000), para que fosse possível comparar variáveis que possuíam unidades com as escalas de variação diferentes.

No programa ISATIS (GEOVARIANCES, 2001), foram calculados variogramas experimentais, para cada variável, e ajustados a modelos teóricos juntamente com variogramas cruzados para os conjuntos de dados em análise. Foram ajustadas três estruturas básicas modeladas por uma combinação linear dos variogramas, padronizados para um mesmo alcance. Com isso, puderam-se verificar as correlações existentes entre as variáveis e a permissibilidade do modelo linear de corregionalização (GOOVAERTS, 1997).

\section{Krigagem fatorial e o modelo linear de corregionalização (MLC)}

A krigagem fatorial é um método geoestatístico para analisar conjuntos de dados espaciais multivariados. A teoria por trás desse método foi descrita em diversos artigos (GOULARD \& VOLTZ, 1992; GOOVAERTS, 1992; CASTRIGNANÒ et al., 2000; LARK \& PAPRITZ, 2003; MANZIONE etal., 2005) e livros texto (JOURNEL \& HUIJBREGTS, 1978; GOOVAERTS, 1997; WACKERNAGEL, 2003) Neste artigo serão descritas apenas as etapas básicas, como se segue.

O modelo linear de corregionalização é o modelo mais comumente usado para estimar a corregionalização. Journel \& Huijbregts (1978) consideram todas $p$ variáveis envolvidas em determinado processo físico, atuando em diferentes escalas espaciais $N_{s}$, para cálculo de $p(p+1) / 2$ variogramas experimentais diretos $\mathrm{e}$ cruzados. Esses variogramas são ajustados como combinações lineares do mesmo conjunto de $N_{s}$ funções variograma básicas $g^{u}(\boldsymbol{h})$ : 


$$
\gamma_{i j}(\mathbf{h})=\sum_{u=1}^{N s} b_{i j}^{u} g^{u}(\mathbf{h}) \quad i, j=1, \ldots, p
$$

em que

$\gamma_{\mathrm{ij}}(\mathbf{h})$ é o modelo de variograma cruzado entre as variáveis $Z_{i}$ and $Z_{j}$

$u$ é a escala espacial;

$\boldsymbol{h}$ é o vetor distância (passo), e

$b_{j}^{u}$ são os coeficientes da função $g^{u}(\boldsymbol{h})$.

Usando-se notação matricial, o MLC pode ser reescrito da seguinte forma:

$$
\Gamma(\mathbf{h})=\sum_{u=1}^{N s} \mathbf{B}^{u} g^{u}(\mathbf{h})
$$

em que

$\boldsymbol{\Gamma}(\boldsymbol{h})$ é a matriz simétrica $p \times p$ cujos valores dos variogramas diretos e cruzados, respectivamente, para uma determinada distância $\boldsymbol{h}$; e

$\mathbf{B}^{u}$ é a matriz simétrica $p \times p$ dos coeficientes $b_{j}^{u}$, chamada matriz de corregionalização.

No MLC, supõe-se que o comportamento espacial das variáveis é resultado da superposição de diferentes processos independentes, atuando em diferentes escalas.

Quando se trata de solos, esses processos podem ser fatores de formação do solo (material de origem, topografia, vegetação, clima e tempo), práticas de manejo (tipo de preparo do solo, rotação de culturas e fertilização) e erosão. Esses processos alteram o comportamento dos variogramas experimentais, que podem ser modelados por um conjunto de funções $g^{u}(\mathbf{h})$. A escolha do número e das características (modelo, patamar, alcance) das funções variograma $g^{u}(\mathbf{h})$ é um tanto delicada e pode ser facilitada por um bom conhecimento do fenômeno em estudo (MANZIONE et al., 2005).

O ajuste do MLC pode ser feito por mínimos quadrados, sob a restrição de que a matriz $\mathbf{B}^{u}$ tem que ser semidefinida positiva. O melhor modelo pode ser escolhido como sugerido por Goulard \& Voltz (1992), comparando-se o ajuste de diversas combinações das funções $g^{u}(\mathbf{h})$ com diferentes alcances, em termos da soma do quadrado dos resíduos. Lark \& Papritz (2003) sugerem a simulação annealing como um método automático para estimar as funções variogramas, minimizando a soma dos quadrados ponderados entre os variogramas ajustados e modelados.

\section{Analisando a estrutura de correlação entre variáveis, aplicando análise de componentes principais (ACP)}

Métodos estatísticos multivariados clássicos podem ser aplicados às matrizes $\mathbf{V}, \boldsymbol{\Gamma}(\mathbf{h})$ e $\mathbf{B}^{u}$. Entre as técnicas descritas em Wackernagel et al. (1989), a análise de componentes principais é geralmente usada para decompor as matrizes de corregionalização $\mathbf{B}^{u} \mathrm{em}$ matrizes $\mathbf{A}^{u}$ e para resumir a relação entre as variáveis. Assim, são geradas novas variáveis ortogonais, os componentes principais, que são combinações lineares das variáveis originais, com coeficientes contidos nos autovetores da matriz (GOOVAERTS, 1992). Cada componente principal explica a percentagem da variância total correspondente à razão do autovalor associado ao traço da matriz. As primeiras componentes, geralmente, resumem a maior parte da variância e, consequentemente, são as mais informativas.

A matriz de variância-covariância clássica é uma combinação de matrizes de corregionalização, relativas a diferentes escalas. Entretanto, quando a correlação entre as variáveis muda conforme a escala espacial, a abordagem clássica da matriz de variância-covariância não descreve de forma apropriada essas correlações. A krigagem fatorial considera a correlação entre as variáveis, em cada escala espacial modelada por meio das matrizes de corregionalização $\mathbf{B}^{u}$. Conforme Goovaerts (1992), a análise de componentes principais clássica reúne as combinações das principais relações entre variáveis em diferentes escalas, enquanto a abordagem geoestatística aumenta o poder exploratório das análises fatoriais, pois permite a distinção desses fatores, de acordo com suas variações em escalas espaciais.

\section{RESULTADOS E DISCUSSÃO}

A análise exploratória dos dados permite um melhor entendimento do conjunto de dados,

\section{REVENG}


descrevendo suas variações e comportamentos em torno da média. Foram analisadas, como medidas de posição, as médias aritméticas das variáveis; como medidas de dispersão em torno da média, a variância, o desvio padrão e o coeficiente de variação e, como medidas de forma, os coeficientes de assimetria e curtose, presentes no Quadro 1.

Os coeficientes de variação fornecem, segundo Landim (1998), uma medida relativa da precisão do experimento, sendo bastante útil na avaliação da dispersão dos dados, normalmente apresentados em percentagem. Considerando os parâmetros propostos por Gomes (1976), a MO apresentou médios coeficientes de variação (entre 10 e $20 \%$ ) e o P e o K apresentaram coeficientes muito altos (> $30 \%$ ). No caso, grandes coeficientes de variação podem demonstrar grandes alterações provocadas pelo homem, como adubações e calagens sucessivas e irregulares, ou, mesmo, local de amostragem em linha ou entrelinha de cultivo. A distribuição de frequência de $\mathrm{P}$ e $\mathrm{K}$ também mostram forte assimetria, decorrente de apresentarem poucos valores altos e muitos valores baixos, atribuindo uma distribuição normal muito assimétrica, positivamente, para $\mathrm{K}$ e distribuição log-normal para P. Para avaliar a normalidade do conjunto de dados, o teste proposto por D'Agostinho (PARKIN \& ROBINSON, 1992) foi realizado, a $5 \%$ de probabilidade. Confirmada a distribuição log-normal para $\mathrm{P}$, verificou-se então a presença de dados discrepantes (ouliers), dentro dos conjuntos de dados de cada variável, sendo para $\mathrm{MO}$, em um ponto, e para $\mathrm{P}$, em nove pontos, dos 204 pontos amostrados. Para a verificação de outliers calcularam-se medidas de dispersão adicionais, analisados os quartis superiores e inferiores das frequências, e, com isso, puderam- se identificar valores discrepantes (HOAGLIN et. al., 1992), resultantes possivelmente de práticas inadequadas de manejo da fertilidade do solo, ou, mesmo, de erros analíticos em laboratório. Eliminando-se esses pontos, as distribuições passaram a apresentar distribuição normal, condição essencial para que pudessem ser modeladas segundo o modelo linear de corregionalização. Também foi necessário padronizar as variáveis, para que ficassem dentro de uma mesma escala de variação e pudessem ter seus comportamentos espaciais comparados.

\section{Análise de corregionalização}

No conjunto de variáveis estudadas para aplicação de fertilizantes, MO, P e K, foram combinadas e ajustadas, simultaneamente, para os variogramas e variogramas cruzados, três estruturas básicas omnidirecionais; a primeira relativa ao efeito pepita; a segunda, um modelo esférico com alcance de $120,18 \mathrm{~m}$ e, a terceira, um modelo exponencial com alcance de 320,18 m. A combinação dessas estruturas, ajustadas no contexto do modelo linear de corregionalização, dividiu a variação dos dados em uma microescala, relativa ao efeito pepita; uma média escala, até $120,18 \mathrm{~m}$, e uma longa escala, até $320,18 \mathrm{~m}$. A Figura 2 exibe os variogramas diretos e cruzados ajustados para o conjunto $\mathrm{P}, \mathrm{K}$ e MO.

A matriz de corregionalização, fornecida pelo programa após os ajustes dos variogramas (Quadro 2), revelou a maior influência do elemento $P$ no comportamento espacial, nas duas primeiras estruturas combinadas, já que seus coeficientes de covariância são superiores em relação aos outros. $\mathrm{Na}$ terceira estrutura, essa influência é dividida com o K.

Quadro 1. Análise estatística exploratória para as variáveis em estudo P, K e MO

\begin{tabular}{ccccccccc}
\hline & & Desvio & \multicolumn{3}{c}{ Coef. de } & Coef. de & Coef. de & \multicolumn{2}{c}{ Distrib. de } \\
Variável & Média & padrão & Variância & Assimetria & Curtose & Variação & Y & Frequência \\
\hline P & 33,51 & 29,88 & 893,12 & 3,12 & 16,72 & 89 & $-29,17$ & LN \\
K & 1,76 & 1,04 & 1,07 & 1,32 & 4,69 & 58 & $-9,17$ & N \\
MO & 24,36 & 2,68 & 7,16 & 0,55 & 6,79 & 11 & 0,48 & N \\
\hline
\end{tabular}

Unidades utilizadas: $\mathrm{P}-\mathrm{mg} \mathrm{dm}{ }^{-3} ; \mathrm{K}-\mathrm{mmol}_{\mathrm{c}} \mathrm{dm}^{-3} ; \mathrm{MO}-\mathrm{g} \mathrm{dm}^{-3}$; Coef. de Variação $=\% ; \mathrm{Y}=$ Coef. de D' Agostinho a ( $5 \%$ de probabilidade); N-distribuição normal; LN- distribuição lognormal. 

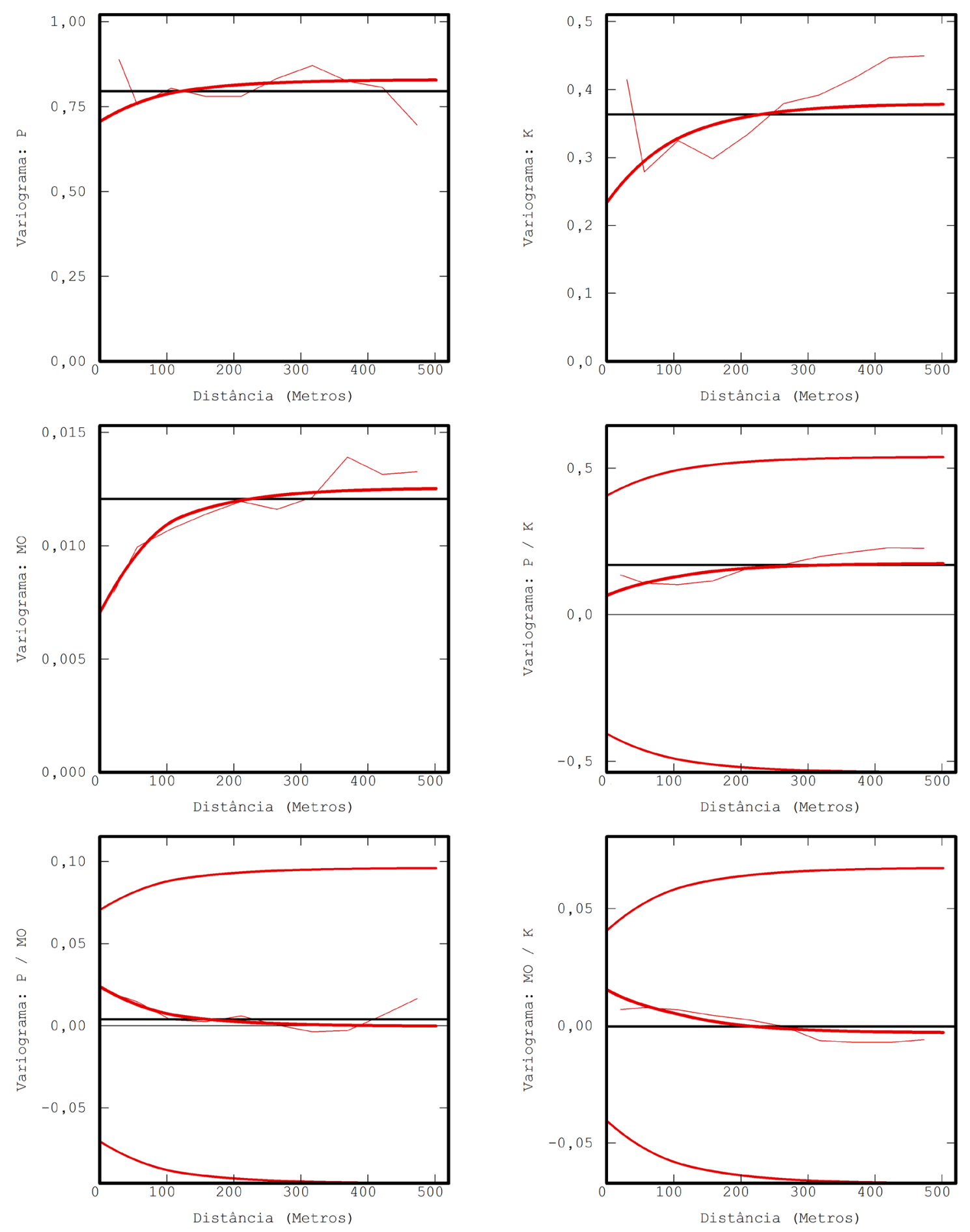

Figura 2. Variogramas, teóricos diretos e cruzados, para os valores padronizados das variáveis P, K e MO. 
Quadro 2. Matriz de corregionalização (coeficientes de covariância) para cada escala espacial do conjunto de variáveis $\mathrm{P}, \mathrm{K}$ e MO

\begin{tabular}{llccc}
\hline & & $\mathrm{P}$ & $\mathrm{K}$ & $\mathrm{MO}$ \\
\hline Micro & $\mathrm{P}$ & 0,7045 & - & - \\
escala & $\mathrm{K}$ & 0,0640 & 0,2119 & - \\
Efeito Pepita & MO & 0,0241 & 0,0156 & 0,0070 \\
Média & $\mathrm{P}$ & 0,0169 & - & - \\
escala & $\mathrm{K}$ & $-0,0114$ & 0,0076 & - \\
$120,18 \mathrm{~m}$ & $\mathrm{MO}$ & $-0,0051$ & 0,0034 & 0,0015 \\
Longa & $\mathrm{P}$ & 0,1071 & - & - \\
escala & $\mathrm{K}$ & 0,1216 & 0,1648 & - \\
$320,18 \mathrm{~m}$ & MO & $-0,0193$ & $-0,0219$ & 0,0040 \\
\hline
\end{tabular}

Quadro 3. Composição estrutural dos dois primeiros componentes principais $(\mathrm{CP})$ para cada escala espacial do conjunto de variáveis $\mathrm{P}, \mathrm{K}$ e MO

\begin{tabular}{llcc}
\hline & & CP1 & CP2 \\
\hline Micro & $\mathrm{P}$ & $-0,8373$ & $-0,0587$ \\
escala & $\mathrm{K}$ & $-0,1078$ & 0,4475 \\
Efeito Pepita & MO & $-0,0310$ & 0,0282 \\
Média & $\mathrm{P}$ & 0,1302 & 0,0000 \\
escala & $\mathrm{K}$ & $-0,0873$ & 0,0000 \\
$120,18 \mathrm{~m}$ & MO & $-0,0388$ & 0,0000 \\
Longa & $\mathrm{P}$ & 0,3170 & 0,0813 \\
escala & $\mathrm{K}$ & 0,4005 & $-0,0665$ \\
$320,18 \mathrm{~m}$ & MO & $-0,0572$ & $-0,0148$ \\
\hline
\end{tabular}

Quadro 4. Composição estrutural dos dois primeiros componentes principais (Autovalores e Autovetores) para cada escala espacial do conjunto de variáveis $\mathrm{P}, \mathrm{K}$ e MO

\begin{tabular}{llcc}
\hline & & Autovetor 1 & Autovetor 2 \\
\hline Micro & $\mathrm{P}$ & $-0,9911$ & $-0,1297$ \\
escala & $\mathrm{K}$ & $-0,1276$ & 0,9896 \\
Efeito Pepita & MO & $-0,0366$ & 0,0624 \\
& Autovalores & 0,7132 & 0,2045 \\
Média & $\mathrm{P}$ & 0,8062 & 0,2858 \\
escala & $\mathrm{K}$ & $-0,5406$ & 0,0000 \\
$120,18 \mathrm{~m}$ & MO & $-0,2404$ & 0,9583 \\
& Autovalores & 0,0261 & 0,0000 \\
Longa & $\mathrm{P}$ & 0,6167 & 0,7666 \\
escala & $\mathrm{K}$ & 0,7792 & $-0,6267$ \\
$320,18 \mathrm{~m}$ & MO & $-0,1114$ & $-0,1400$ \\
& Autovalores & 0,2642 & 0,0112 \\
\hline
\end{tabular}

O comportamento de $\mathrm{P}$ e $\mathrm{K}$ foi de pouca variabilidade, quando comparado com o de MO, interferiu na modelagem da corregionalização, como pode ser visto na decomposição das variáveis em fatores regionalizados (Quadro 3).

Essa interferência foi expressa pelos autovetores e autovalores dos componentes principais (Quadro 4), em que a estrutura do $\mathrm{P}$ carrega a maior variação, em função das variáveis originais, dividindo com o $\mathrm{K}$ essa influência, na última parte do modelo teórico, apesar das baixas correlações existentes. 
Nota-se que, para a segunda estrutura, de modelo esférico, com alcance de 120,18 m, e para estrutura exponencial, com alcance de $320,18 \mathrm{~m}$, os autovalores diminuem muito em relação ao efeito pepita e são quase nulos para os outros componentes espaciais, ficando evidente descontinuidades desde a origem dos variogramas. Os variogramas de $\mathrm{P}$ e $\mathrm{K}$ poderiam ser interpretados, praticamente, como um efeito pepita puro, se analisados individualmente, por causa da baixa variância da amostra. Isso se dá por causa dos baixos valores dos elementos encontrados na área, decorrente do consumo dos nutrientes pelos cultivos. Outro fator que contribui para esse comportamento é o fato de a amostragem não ter distinguido linhas e entrelinhas de cultivo, fazendo com que, em alguns casos, houvesse realmente valores discrepantes. No caso da MO, os valores baixos são decorrentes das características naturais desses solos, do clima da região, que favorece a atividade microbiológica do solo, que consome a maior parte desse conteúdo, além do histórico de adubações químicas na área, que acaba sendo a grande responsável pela suplementação nutricional das culturas ali instaladas em rotação, no caso, soja e milho. De forma geral, a utilização da análise de corregionalização como ferramenta geoestatística multivariada revelou, de forma eficaz, o comportamento das variáveis no solo para as escalas propostas. A análise conjunta das variáveis, envolvidas no processo de adubação da cultura, permite visualizar comportamentos que seriam perdidos, se analisados individualmente.

A análise de corregionalização, ao modelar diferentes escalas espaciais, forneceu informações sobre possíveis zonas de manejo, para adubações a taxas variáveis. Pode-se dizer que um equipamento de agricultura de precisão, que fosse trabalhar nessa área, precisaria ter uma resolução espacial de no mínimo $320,18 \mathrm{~m}$, para que pudesse trabalhar as variações espaciais que ocorrem ali. Entendese essa resolução espacial como a distância que o veículo percorre entre um ponto e outro do mapa, com tempo suficiente para variar a taxa de aplicação. Caso o equipamento não opere em escalas menores, recomenda-se ajustar a formulação dos adubos pelo elemento que mais influencia o processo, no nosso caso, o Fósforo (P).

\section{CONCLUSÕES}

- A análise especial multivariada apresentada mostrou-se uma ferramenta adequada para explorar a estrutura espacial, em diferentes escalas, de um conjunto de dados relacionados com um problema específico, ao unir técnicas geoestatísticas e análise de componentes principais;

- Para o conjunto de dados P, K e MO, o P exerce maior influência em microescala e média escala (120,18 m), enquanto, em longa escala $(320,18$ $\mathrm{m})$, essa influência é dividida com o K; e

- É possível, a partir das escalas modeladas, estabelecer zonas de manejo para aplicação de fertilizantes, formulados a partir de $\mathrm{P}$ e $\mathrm{K}$, respeitando a variabilidade espacial dos elementos no solo.

\section{AGRADECIMENTOS}

À FAPESP (Processo \# 2000/05515-9), pelo auxilio financeiro na execução desse projeto. Ao Eng $^{\circ}$ Agr $^{\circ}$ Antonio Carlos Menezes de Mendonça, por permitir os estudos em sua propriedade. Ao Eng $^{\circ}$ Agro $^{\circ}$, M.Sc., João Batista Tolentino Rodrigues, a João Vieira de Andrade e a Fábio Vieira de Andrade, pelo auxílio nas amostragens e demais atividades de campo.

\section{REFERÊNCIAS BIBLIOGRÁFICAS}

BLACKMORE, S. The interpretation of trends from multiple yield maps. Computers and eletronics in agriculture, v.26, n 1, p.37-51, 2000.

BURROUGH, P.A.; BOUMA, J.; YATES, S.R. The state of the art in pedometrics. Geoderma, v.62, n.1-3, p.311-26, 1994.

CASTRIGNANÒ, A.; GIUGLIARINI, L.; RISALITI, R.; MARTINELLI, N. Study of spatial relationships among some soil physicochemical properties of a field in central Italy using multivariate geostatistics. Geoderma, v.97, n.1-2, p.39-60, 2000. 
EMBRAPA. Empresa Brasileira de Pesquisa Agropecuária. Manual de métodos de análise de solo. $2^{a}$ edição. Rio de Janeiro,: CNPS/ EMBRAPA, 1997. 212p.

EMBRAPA. Empresa Brasileira de Pesquisa Agropecuária. Classificação brasileira de solos. $1^{a}$ edição. Rio de Janeiro:CNPS/ EMBRAPA, 1999. $412 p$.

GEOVARIANCES. ISATIS 3.3. Software Manual, Avon, France, 2001. 531 p.

GOMES, F.P. Curso de estatistica experimental. Piracicaba: Ed. CALQ/ESALQ, 1976. 430 p.

GOOVAERTS, P. Factorial kriging analysis: a useful tool for exploring the structure of multivariate spatial soil information. Journal of Soil Science, v.43, n.4, p.597-619, 1992.

GOOVAERTS, P. Geostatistics for natural resources evaluation. Oxford: Oxford press. 1997. 483p.

GOULARD, M.; VOLTZ, M. Linear coregionalization model: Tools for estimation and choice of cross-variogram matrix. Mathematical Geology, v.24, n.3, p.269-86, 1992.

HOAGLIN, D.C.; MOSTELlER, F.; TUKEY, J.W. Análise exploratória de dados - Técnicas robustas. Um guia. Série Novas Tecnologias. Lisboa: Ed. Salamandra. 1992. 417p.

JOURNEL, A.G.; HUIJBREGTS, C.J. Mining geostatistics. London: Academic Press, 1978. 600p.

LANDIM, P.M.B. Análise estatística de dados geológicos. São Paulo: Ed.UNESP, 1998. 226p.
LARK, R.M.; PAPRITZ, A. Fitting a linear model of coregionalization for soil properties using simulated annealing. Geoderma, v.115, n.3-4, p.245-260, 2003.

MANZIONE, R.L., CAMARA, G. MONTEIRO, A.M.V., ZIMBACK, C.R.L., DRUCK, S. Exploring the multivariate spatial structure of soil acidity data. In: RENARD, P. et al. (Ed.) Geostatistics for Environmental Applications. Berlin: Springer, 2005. (with CD-ROM)

PARKIN, T.B.; ROBINSON, J.A. Analysis of lognormal data. In: STEWART, B.A. (Ed.). Advances in Soil Science. New York: SpringerVerlag, 1992. v.20, p.191-235.

VIEIRA, S.R. Geoestatística em estudos de variabilidade espacial do solo. In: NOVAIS, R. F.; ALVAREZ V, V.H.; SCHAEFER, C.E.G.R. Tópicos em ciência do solo. Viçosa: Sociedade Brasileira de Ciência de Solo, 2000. v.1, p.1-54.

WACKERNAGEL, H.; PETITGAS, P.; TOUFFAIT, Y. Overview of methods for corregionalization analysis. In: ARMSTRONG, M. (ed) Geostatistics. Dordrecht: Kluver Academic Publishers, 1989. v.1, p.409-420.

WACKERNAGEL, H. Multivariate Geoestatistics - An introduction with applications. Berlin: Springer-Velag, 2003. 403p.

WEBSTER, R. Quantitative spatial analysis of soil in the field. In: STEWART, B.A. (Ed). Advances in soil science. New York: Springer-Verlag, 1985. v.3, p.1-70.

WOLLENHAUPT, N. Q \& A with a professor: smaller grids pay. Dealer Progress, v.25, n.7, p.64-68, 1995. 